\title{
Recombinant Interleukin-17
}

National Cancer Institute

\section{Source}

National Cancer Institute. Recombinant Interleukin-17. NCI Thesaurus. Code C1684.

A recombinant agent, which is chemically identical or similar to the endogenous cytokine interleukin-17 (IL-17). IL-17 is produced by cytotoxic T cells and stimulates stromal cells to produce pro-inflammatory and hematopoietic cytokines. This cytokine enhances expression of IL-6, IL-8, and ICAM-1 in fibroblasts and appears to induce osteoclastogenesis. 